Das Dibromid krystallisirt aus Alkohol in bei $192^{\circ}$ schmelzenden Nadeln.

$0,1596 \mathrm{~g}$ gaben $0,1408 \mathrm{AgBr}$.

Berechnet für Gefunden

$\mathrm{Br}$

$$
\mathrm{O}_{18} \mathrm{H}_{20} \mathrm{O}_{2} \mathrm{Br}_{2}
$$

37,54

Ueber die Constitution des Dimethylpiperidins und seiner Homologen;

ron A. Ladenburg, M. Mugdan und O. Brastoricz; mitgetheilt von $A$. Ladenburg.

(Eingelaufen am 22. Mai 1894.)

Bekanntlich liat A. W. v. Hofmann gezeigt, dass die Methylirung des Piperidins den ron ilm vordem aufgestellten Regeln zuwiderläuft, indem das Piperidin, welches zweifellos eine secundäre Base ist, zwei Atome Wasserstoff gegen Mrethyl austauscht und so das unzersetzt flüchtige Dimethylpiperidin $\mathrm{C}_{5} \mathrm{H}_{9} \mathrm{~N}\left(\mathrm{CH}_{3}\right)_{2}$ liefert. Die Erklärung dieser bemerkenswerthen Thatsache glaubte Hofmann dadurch geben zu können, dass er annahm, das zweite Methyl sei in den Kern getreten, d. b. an Kohlenstof gebunden und er gab daher dem Dimethylpiperidin etwa folgende Formel:

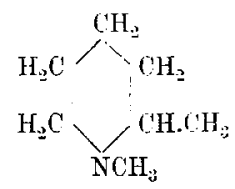

Ich habe dagegen ausgeführt, dass diese Formel unmöglich richtig sein könne, weil sonst das Dimethylpiperidin mit einem methylirten Pipecolin identisch sein müsse, was thatsächlich nicht der Fall ist, und weil auch das ganze Verhalten des Dimethylpiperidins gegen die H ofman n'sche Auffassung spricht. Ich schlug die folgende Formel vor: 


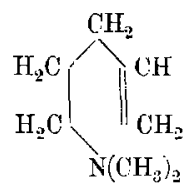

welche namentlich auch für die, bei der weiteren Einführung von Methyl, erfolgende Spaltung in Trimethylamin und Piperylen $\mathrm{C}_{5} \mathrm{H}_{8}$ ein Verstïndniss bot.

Merling, der anfangs gegen diese letztere Auffassung aufgetreten ist ${ }^{1}$ ), hat später neve Argumente für sie beigebracht.

Hofmann hatte nämlich weiter angegeben, dass das Dimethylpiperidin durch Erwärmen im Salzsäurestrom unter Abspaltung von Chlormethyl Methylpiperidin regenerire. Merling zeigt nun ${ }^{2}$ ), dass diese Augabe unrichtig ist, indem nicht Methylpiperidin, sondern eine davon rerschiedene Base, die Meriing als Dimethylpyrrolidin auffasst, entsteht. Er zeigt ferner, dass dicse Zersetzung in der Art vor sich geht, dass zunächst zwei Moleküle Salzsäure addirt werden, wodurch salzsaures Hydrochlordimethylpiperidin entsteht.<smiles>CCCCCC(C)C(C)Cl</smiles>

Die daraus durch Natronlauge abgeschiedene Base, das Hydrochlordimethylpiperidin, verwandelt sich beim Erhitzen auf $150^{\circ}$ in das isomere Dimethylpyrrolidinmethylammoniumehlorid<smiles>CCC(C)C(C)CCl</smiles>

welches bei weiterem Erhitzen Chlormethyl abspaltet und ra-Dimethylpyrrolidin erzeugt.

Ausserdem wird in der angezogenen Untersuchung nachgewiesen, dass Dimethyl- $\alpha$-Pipecolin sich gegen Salzsäurc

1) Ber. d. deutsch. chem. Ges. 17, 2139.

2) Diese Amulen 264, 310 . 
ganz anders verhält, indem es nicht in eine Pyrrolidinverbindung übergeht, sondern $\boldsymbol{r}$-Methyl- $\boldsymbol{x}$-Pipecolin erzeugt, was Merling in folgender Weise zu erkiären versucht:<smiles>CC(C)C1CCCC1</smiles>

Dimethyl- $\epsilon$-Tipecolin ammoniumjodid<smiles>CCCCC(C)C(C)C</smiles>

Eydrochlordimethy' $\alpha$ - Pipeculin<smiles>C=C(C)C(C)CC</smiles>

Ijimethyl-a- P'ipecolin

(Butallylearbindimethylamin)

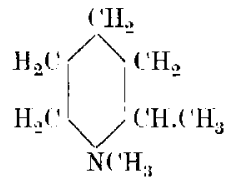

v-Methyl-«-Pipecolin.

Dagegen findet Merling, dass das von ihm synthetisch gewonnene Butallylmethylcarbiadimethylamin sich wie Dimethylpiperidin verhält, d. h. bei der Behandlung mit HCl etc. ein Pyrrolidinderivat, nämlich Trimethylpyrrolidin erzeugt, was durch folgende Formeln anschaulich zu machen versucht wird:

$$
\mathrm{CH}_{8} \text { : (H.CH.CH.CII.N(C) }
$$

Butallylmethylearbindimethylamiu,

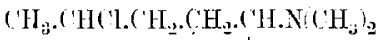

$$
\begin{aligned}
& \mathrm{CH}_{3}
\end{aligned}
$$

Hydrochlorbutallylmethylcarbindimethylamiv,

$$
\text { - Methyl-ces- I)imethylpyrolidin. }
$$

Aus diesen verschiedenen Thatsachen glaubt nun Merling schliessen zu dürfen, dass nur diejenigen Basen, welche die Gruppe 
<smiles>[R1][CH][AlH][CH]</smiles>

enthalten, sich wie $v$-Methyl- $\iota$-Pipecolin bei Methylirung und nachheriger Behandlung mit $\mathrm{HCl}$ verhalten und schliessiich die Ausgangsbase regeneriren.

Dieser Schluss, der zunächst noch mit einer gewissen Zurïckhaltung ausgesprochen wird, erlangt für Merling nach einigen Monaten, ohne dass neue Thatsachen zu seiner Begründung beigebracht werden, apodiktische Gewissheit, und wird benutzt, um daraus sehr eigenthümliche Formeln für Tropin und Ecgonin aufzustellen ${ }^{3}$ ).

Gegen diese Auffassungen bin ich bereits vor längerer Zeit aufgetreten, indem ich namentlich hervorhob, dass der Satz, wonach nur diejenigen Piperidinderivate mit der Gruppe<smiles>CCC(C)[AlH2+]</smiles>

bei Methylirung und Salzsüurebehandlung regenerirt würden, weit über die Thatsachen limansgehe, und darthat, dass alle bisher beobachteten hierher gehörigen Erscheinungen zu der Annahme führen, dass $\alpha$-substituirte Piperidine bei jener Behandlung regenerirt würden, während Piperidin selbst und andere Piperidinabkömmlinge zu Pyrrolidinderivaten führen ${ }^{4}$ ).

Obgleich nun spätere Versuche von Merling damit vollkommen im Einklang standen $^{5}$ ), so habe ich doch geglaubt, auch meinerseits diese viel umstrittene Frage durch weitere Versuche ihrer Lösung näher zu bringen. Und zwar wurden diese Versuche in erster Linie auf das Coniin ausgedehnt.

Hier aber hat eine Beobachtung ron grosser Tragweite, welche der Eine ron uns (Mugdan) machte, die ganze Frage

:) Ber. d. deutsch. ehem. Ges. 24, 3108 .

4) Ber. d. deutsch. chem. Ges. $\mathbf{2 6}, 1064$.

5) Diese Annalen 278, 1. 
in eine neue Phase geruclit, sodass die Versuche einen grösseren Umfang anmahmen als Anfangs projectirt war.

Es hat sich nämlich gezeigt, dass das Dimetlylconion optisch activ ist, während weder Merling's Formel für Dimethylpipecolin, noch die meinige zu einem Gebilde mit asymmetrischem kohlenstoff führt:

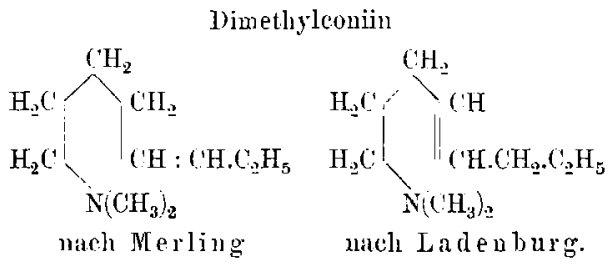

Man könnte nun versucht sein, um den asymmetrischen Kohlenstofi des Coniins zu erhalten, dem Dimethylconiin folgende Formel zu geben:<smiles>C=CCC(CCCC)C(C)C</smiles>

allein dann hätte es bei der Behandlung mit $\mathrm{HCl}$ sich wie Dimethylpiperidin verhalten und ein Pyrrolidinderivat liefern müssen, wenn überhaupt die Constitution des Dimethylpiperidins richtig angenommen war. So waren denn jetzt die Spaltungsversuche des Dimetlyylconiins von besonderer Bedeutung geworden. Sie lieferten, wie unten gezeigt wird, Coniin. Feruer schien es angezeigt, die Zerlegung des Dimethylpiperidins einer erneuten Prüfung $\mathrm{zu}$ nuterwerfen und womöglich den bestimmten Nachweis zu führen, dass dieselbe zu einer Pyrrolidinverbindung führe, indem man entweder bis zum Pyrrolidin selbst oder bis zu einem bekannten Derivate den Abbau fortsetzte. Auch lieses ist gelungen, und damit war die obige Formel für Dimethylconiin als unrichtig zu verwerfen. Es blieb uns dann noch die Formel übrig 
deun auch die Formel<smiles>CCCC1CCC2(CCCC2)[C@H]1C</smiles>

$$
\mathrm{H}_{2} \mathrm{C} \overbrace{\mathrm{H}_{2}} \mathrm{CCH}_{3})_{2} \cdot \mathrm{CH}_{2} \cdot \mathrm{C}_{2} \mathrm{H}_{5}
$$

ist, weil sie kein asymmetrisches Kohlenstoffatom besitzt, ausgeschlossen.

Weitere Gründe für diese Auffassung des Verhaltens der $\alpha$-substituirten Piperidinderivate lieferten die bereits früher und neuerdings gewomenen Erfahrungen beim $\alpha$-Pipecolin. Hier wurde zunächst aus einem optisch activen Pipecolin Dimethylpipecolin dargestellt und auch dieses optisch activ, wemn auch nur schwach drehend, gefunden. In Folge dessen kounten die früheren Formelı für dasselbe nicht beibehalten werden, da diese nicht asymmetrisch sind. Die dann zunächst ins Auge zu fassende Formel<smiles>CC=CCC(C)C(C)C</smiles>

ist aber aus denselben Gründen wie die entsprechende für das Dimetbylconiiu zu verwerfen, wobei noch als neues und entscheidendes Argument hinzukommt, dass dieses Schema aus triftigen Gränden dem Butallylmethylcarbindimethylamin zugeschrieben wird (siehe oben und diese Annalen $\mathbf{2 6 4}, 310$ ). So bleibt denn für das Dimethyl- $\alpha$-Pipecolin nur eine Formel, wonach es ein Abkömmling eines c-Pentans ist, nämlich

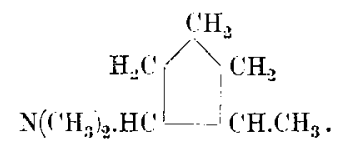


Erscheint so cinerseits, nachdem alle auderen für das Dimethylpipecolin etwa sonst in Betracht kommenden Formeln ausgeschlossen sind, dieses Schema als das einzig mögliche, so kaun man doch andererseits nicht behaupten, dass es mit allen Erfahrungen in guiem Einklang stehe. Schon die hier vielfach erwähnte, leichte Addition von Salzsïure erseheint immerbin auffallend, wenn man die sonst beobachteten Thatsachen über die Stabilität des c-Pentans in Betracht zieht. Es muss ferner hervorgehoben werden, dass die von Merling ${ }^{6}$ ) beobachtete Entstehung von Diallyl aus dieser Base durch dieses Schema eine weit weniger befriedigende Erlilärung findet, als das bei den fruheren Formeln der Fall war.

Wenn diese Gründe auch nicht schwerwiegend genug sind, um die aufgesteliten Formeln zu verwerfen, so sollte doch hier betont werden, dass die Theorie von van t'H off und Le Bel hier zu wirklichen Schwieriglieiten führt, worauf unten zurüclgekommen ist. Ferner soll lier noch hervorgehoben werden, dass die nene Auffassung für das Dimetbylpiperidin dieses als der von $J$. Wislicenus kürzlich ans dem Adipinlieton erhaltenen Base ${ }^{i}$ )<smiles>CC1CCCC1(C)N</smiles>

und dem ron Semmler aus dem Methyladipinketon gewonneneis Methylpentamethenylamin

verwandt erscheinen lïsst.<smiles>CCC(C)CC(C)C</smiles>

Selbstverständlich erscheint jetzt auch dic Zerlegung des Tropidins und die Bildung von Tropiliden in neuem Lichte, weshalb ich noch kurz hicrauf eingehen will.

6) Diese Annalen 264, 310 .

7) Diese Annalen 275, 326i. 
Tropidin behält die für dasselbe schon früher ${ }^{8}$ ) anfgestellte Formel:

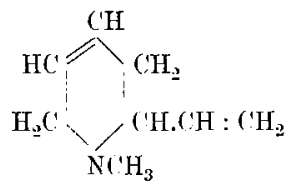

$r$ - und $\beta$-Methyltropidin werden aber jetzt in folgender Weise formulirt:<smiles>C=C1CCC=CC1C</smiles><smiles>CC(C)C1CC=CC1C</smiles>

wobei vorausgesetzt wird, dass sowohl Tropilen wie Tropiliden aus $\beta$-Methyltropidin gebildet werden, während aus der $\alpha$-Verbindung dureh Aufnahme von $\mathrm{HCl}$, wobei der Ring gesprengt wird, unter Abspaltung von $\mathrm{CH}_{3} \mathrm{Cl}$ Tropidin regenerirt wirl. Das Tropiliden behält seine frühere Formel

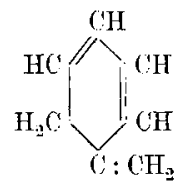

und seine Entstehung aus $\beta$-Methyltropidin ist ohne weiteres zul verstehen.

\section{Experimenteller Thoil.}

\section{Darstellung von Dimethylpiperidin.}

Schon früher habe ich gezeigt, dass beim Erhitzen von salzsaurem Piperidin mit Methylalkohol auf $200^{\circ}$ neben $y$-Methylpiperidin Dimethylpiperidin entsteht $\left.{ }^{9}\right)$. Jetzt wurde diese Reaction zur Darstellung des letzteren Köruers benutzt.

8) ber. d. deutsch. chem. Ges. 2B, 1067.

9) Ber. d. deutsch. chem. Ges. 16, 20 ǒ7. 
Es werden je $10 \mathrm{~g}$ Piperidinchlorhydrat mit $10 \mathrm{~g}$ absolutem Methylalkohol in zugeschmolzenem Robre zehn Stunden lang auf $250^{\circ}$ erhitzt. Der Röhreninhalt stellt einen zähen Syrup dar, auf dem cine kleine Schicht Chlormethyl schwimmt, weshalb die Röhren mit grosser Vorsicht geöfinet werden müssen. Der Syrup wird mit Wasser aufgenommen und der überschüssige Methylalkohol durch Abdampfen entfernt. Der Rückstand wurde darauf mit Chloroform ausgeschüttelt, im kleine Mengen harziger Beimengungen zu entfermen, alsdanu wurde mit Natron versetzt und die geringen Mengen von Methylpiperidin abdestillirt. Nach dem Erkalten wurde mit Salzsäure neutralisirt und eingedampft. Dem Rückstand wurde das Dimethylpiperidinammoniumchlorid durch Alkohol entzogen, wobei Kochsalz zurückbleibt. Die Reinheit des so gewonnenen Ammoniumchlorids wurde durch Darstellung des Platindoppelsalzes und Golddoppelsalzes sicher gestellt. Das erstere: $\left(\mathrm{C}_{7} \mathrm{H}_{16} \mathrm{NCl}\right)_{2} \mathrm{PtCl}_{4}$, wird durch Abdampfen der mit Platinchlorid versetzten Lösung des Ammoniumchlorids und Fällen mit Aetheralkohol gewonnen. Nach dem Umkrystallisiren aus heissem Wasser scheidet sich der Körper in prächtigen, rothen, durchsichtigen Krystallen aus. Die Analyse ergab Folgendes:

$0,2438 \mathrm{~g}$ gaben 0,0744 Pt.

$\begin{array}{ccc}0,1476 \mathrm{~g} & , \quad 0,1434 \mathrm{CO}_{2} \text { und } 0,1567 \mathrm{H}_{2} \mathrm{O} . \\ & \text { Gerechnet firr } & \text { Gefunden } \\ & \left(\mathrm{C}_{7} \mathrm{H}_{16} \mathrm{NCl}\right)_{2} \mathrm{P}+\left(\mathrm{l}_{4}\right. & \\ \mathrm{Pt} & 30,59 & 30,52 \\ \mathrm{C} & 26,44 & 26,50 \\ \mathrm{H} & 5,04 & 5,21\end{array}$

Das Goldsalz, $\mathrm{C}_{7} \mathrm{H}_{16} \mathrm{NHCl}_{\mathrm{AuCl}}$, fällt beim Versetzen des Ammoniumchlorids mit Goldchlorid als hellgelber Niederschlag aus, der aus heisser Salzsäure umlrrystallisirt in feinen, gelben Nadeln gewonnen wird.

0,2393 in des trocknen Salzes ginbeu 0,1039 An.

Berechnet fïr Gefiruden $\mathrm{C}_{6} \mathrm{H}_{10} \mathrm{~N}\left(\mathrm{LAuCl}_{3}\right.$

$\mathrm{Au} \quad \mathbf{4 3 , 4 6}$ 
Von diesem Ammoniumchlorid wurden circa $65 \mathrm{pC}$. der theoretischen Ausbeute erhalten. Zur Darstellung von Dimethylpiperidin wurde das Chlorid in Wasser gelöst, mit einem Ueberschuss ron frisch gefälltem Silberoxyd zusammengerührt, wobei die Umwandlung fast momentan vor sich geht. Die vom Chlorsilber filtrirte Flüssigkeit wurde auf dem Wasserbade ziemlich weit eingedampft und trocken destillirt. Dabei zeigte sich ein solches Schäumen, Blasenwerfen, Stossen und dergleichen, dass zum Abdestilliren weniger Cubikcentimeter Stunden erforderlich waren. Alle bekannten Mittel, diesem Uebelstande zu begegnen, schlugen fehl. Schliesslich zeigte sich, dass durch Anwendung einer njcht zu engen Capillare, mittelst welcher Luft auf die Oberfläche geblasen wurde, ein ruhiges Destilliren $z \mathfrak{u}$ erzielen war (B). Aus dem Destillat wurde die Base durch Kali abgeschieden und über Kali getrocknet. Sie siedete bei $117-120^{\circ}$.

\section{Darstellung ron Dimethylpyrrolidin.}

Dieselbe wurde zum Theil nach der von Merling angegebenen Methode ausgeführt ${ }^{10}$ ) zum Theil wurde nach einem abgekürzten Verfahren gearbeitet, das hier angegeben werden soll.

In salzsaures Dimethylpiperidin wurde bei einer Temperatur, die bis $220^{\circ}$ stieg, so lange Salzsüuregas eingeleitet, als Chlormethyl entwich. Dann wurde der Salzsäurestrom unterbrochen und weiter erhitzt. Zunächst entwich Chlorwasserstoff, dann trat Zersetzung ein und es destillirte eine Base, deren Platiusalz ans alkoholischer Lösung lirystallisirt erhalten wurde; dasselbe schmolz bei $222-225^{\circ}$ unter Zersetzung.

$0,2563 \mathrm{~g}$ bei $10 \mathrm{G}^{\mathrm{n}}$ getrocknet gaben $0,0818 \mathrm{Pt}$.

$\begin{array}{ccc} & \text { Berechnet tïir } & \text { Gefunden } \\ \text { P. } & \left(\mathrm{C}_{6} \mathrm{H}_{13} \mathrm{NHCl}_{2} \mathrm{PtCl}_{4}\right. & \\ & 32,00 & 31,93\end{array}$

Die Base ist demnach identisch mit Dimethylpyrrolidin. Die Ausbeute war aber keine sehr zufriedenstellende. Der Siedepunkt der Base wurde zu $98-101^{\circ}$, das spec. Gew. bei $0^{0}=$

10) Diese Aunalen 264, 310.

Annalen der Chemie 279. Bu. 
0,8089 , bei $15^{\circ}=0,7968$ gefunden. Merling giebt als Siedepunkt $96-97^{0}$, für das spec. Gew. bei $15^{0}=0,799$ an.

\section{Verwandlung von Dimethylpyrrolidin in Dimethylpiperidin.}

Wird die vorstehend beschriebene Base in methylalkoholischer Lösung mit einem Molekül Jodmethyl tropfenweise und unter Kühlung versetzt und dann bis zum Eintritt neutraler Reaction erwärmt, so erhält man eine Lösung von Trimethylpyrrolidiniumjodür. Dieselbe wird mit Wasser versetzt, der Alkobol durch Abdampfen entfernt und durch Silberoxyd entjodet. Die Lösung der entstandenen Ammoniumbase wird durch Destillation zunächst von Wasser befreit, zuletzt geht mit demselben ein Oel über, das über Kali getrocknet den Siedep. $117-118^{\circ}$ und das spec. Gew. 0,7675 bei $0^{0}$ besass. (Das specifische Gewicht von Dimethylpiperidin wurde zu 0,7705 bei $0^{0}$ gefunden). Da auch die Eigenschaften der Salze mit denen des Dimethylpiperidins übereinstimmen, so ist kein Zweifel, dass hier Dimethylpiperidin entstanden ist.

Die Reaction muss in folgender Weise aufgefasst werden:

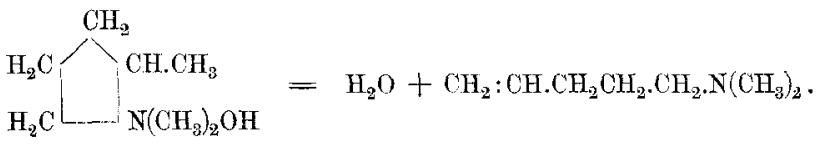

IV. Verwandlung von Dimethylpyrrolidin in a-Methylpyrrolidin.

Wird salzsaures Dimethylpyrrolidin bis auf etwa $220^{\circ}$ erhitzt, indem ein Strom trockner Salzsäure darüber geleitet wird, so spaltet sich reichlich Chlormethyl ab. Nach beendeter Reaction wird das zurückgebliebene Chlorhydrat in Wasser gelöst und in Nitrosamin verwandelt. Dieses in Aether gelöst und über Chlorcalcium getrocknet, lieferte nach der Entfernung des Lösungsmittels ein über $200^{\circ}$ nicht constant siedendes Oel. Bei der Zerlegung mit gasförmiger Salzsäure bei mässiger Temperatur entstanden durch Harzbildung Verluste, so dass nur verhältnissmässig wenig und kein reines Chlorhydrat erhalten wurde. Daher konnte auch weder ein brauchbares Gold- noch 
Platinsalz daraus gewonnen werden und wurde das vorhandene Material zur Darstellung eines Quecksilbersalzes verwerthet, das aus heissem Wasser umkrystallisirt in durchsichtigen, scharf ausgebildeten Prismen erhalten wurde. Nach dem Trocknen bei $100^{\circ}$, wobei kein Krystallwasser entwich, gab dasselbe bei der Analyse folgende Resultate:

\begin{tabular}{ccc}
$0,2555 \mathrm{~g}$ gaben $0,0209 \mathrm{H}_{2} \mathrm{O}$ und $0,0352 \mathrm{CO}_{2} \cdot$ \\
$0,3477 \mathrm{~g} \quad \% \quad 0,2773 \mathrm{HgS.}$ & Gefunden \\
\multicolumn{3}{c}{ Berechnet für } \\
& $\mathrm{C}_{5} \mathrm{H}_{11} \mathrm{NIICl} .5 \mathrm{HgCl}_{2}$ \\
$\mathrm{H}$ & 0,81 & 0,98 \\
$\mathrm{C}$ & 4,06 & 3,76 \\
$\mathrm{Hg}$. & 67,77 & 67,59
\end{tabular}

Bei einer zweiten Darstellung wurde die Reinigung mittelst der Nitrosoverbindung umgangen und aus dem direct erhaltenen Chlorhydrat durch Natronlauge die Base in Freiheit gesetzt und mit Wasser destillirt. Das Destillat wurde mit Salzsäure neutralisirt, abgedampft, in Alkohol gelöst und mit einer alkoholischen Platinchloridlösung versetzt, wodurch sofort ein gelber Niederschlag ausfiel, der mit Aetheralkohol gewaschen wurde. Aus heissem Wasser krystallisirte das salz in feinen Nadeln, die bei $172 \ldots 173^{0}$ schmolzen.

Fine Platimbestimmung des bei $100^{\circ}$ getrockneten Salzes mit $0,2399 \mathrm{~g}$ gab 0,0785 Pt.

$\begin{array}{cc}\text { Berechnet fiur } & \text { Gefurden } \\ \left(\mathrm{C}_{5} \mathrm{H}_{12} \mathrm{NHCl}\right)_{3}, \mathrm{PtCl}_{4} \mathrm{H}_{\mathbf{2}} \mathrm{O} & 32,72 \\ 32,52 & \end{array}$

Genau dieselben Zablen haben Tafel ${ }^{11}$ ) und Neugebauer bei der Analyse des Platindoppelsalzes von $\alpha$-Methylpyrrolidin gefunden, das sie aus dem Anhydrid der $\hat{\gamma}$-Amidovaleriansäure erhielten. Den Schmelzpunkt ihres Platinsalzes geben sie leider nicht an. Wir glauben aber doch aus der sonstigen Uebereinstimmung den Schluss auf die Identität beider Basen ziehen zu sollen. Dass hier kein Piperidin vorliegt, ist sowohl durch

i1) Ber. d. deutsch. chem. Ges. 22, 1866. 
die Eigenschaften dieses Platindoppelsalzes, wie durch die des Chlorhydrats, welches sehr zerfliesslich ist, erwiesen.

Zur weiteren Charakterisirung der entstandenen Base wurde auch noch das Golddoppelsalz dargestellt, das aus der concentrirten Lösung des Chlorhydrats durch Goldchlorid gefällt und aus heissem Wasser umkrystallisirt in hubschen Nadeln erhalten wird. Bei der Goldbestimmung lieferte das Salz Zahlen, die auf das normale Goldsalz $\mathrm{C}_{5} \mathrm{H}_{11} \mathrm{NHClAuCl}_{3}$ gut stimmen.

$0,1282 \mathrm{~g}$ gaben $0,0590 \mathrm{Au}$.
Berechnet
An $\quad 46,32$
Gefunden
46,02

Der Schmelzpunkt dieses Goldsalzes wurde bei $212^{0}$ gefunden.

T afel und Neugebauer scheinen das normale Goldsalz nicht erhalten $z u$ haben. Sie beschreiben wenigstens nur ein Goldsalz, welches aus allroholischer Lösung durch Aether gefällt wird und die sehr unwahrscheinliche Fornel $\left(\mathrm{C}_{6} \mathrm{H}_{11} \mathrm{NHCl}\right)_{2} \mathrm{AuCl}_{3}$ besitzen soll.

\section{Darstellung des Dimethylconiins.}

Man löst $24 \mathrm{~g}$ Coniin in der doppelten Menge Alkohol und versetzt die Lösung mit $25 \mathrm{~g}$ Kali, die in $100 \mathrm{~g}$ Alkohol gelöst sind $\left.{ }^{12}\right)$. Dann wird nach und nach $80 \mathrm{~g}$ Jodmethyl unter Kühlung zugesetzt, wobei ubrigens trotzdem eine starke Wärmeentwickelung zu bemerken ist. Nach beendeter Reaction wird die Masse zur Trockne gedampft, wobei nur wenig Base entweicht, und dem Rückstande durch mehrmaliges Auskochen mit Chloroform das gebildete Dimethylconiumjodür entzogen. Nach Entfernung des Lösungsmittels wird in Wasser gelöst, der Rest des Chloroforms fortgetrieben und die wässrige Lösung zur Krystallisation gebracht, wobei prachtvolle grosse Prismen erhalten werden. Das Jodür wird dann wieder in Wasser gelöst und durch Silberoxyd entjodet, von dem Jodsilber filtrirt, aus der Lösung das meiste Wasser verdampft

\footnotetext{
1.) Vergl. Ia deuburg, diese Amalen $\mathbf{2 4 7}, 56$.
} 
und zuletzt destillirt, wobei schliesslich Dimethylconiin in reichlicher Menge (75 pC. der Theorie) erhalten wird. Die Base siedet nach dem Trocknen, den Hofmann'schen Angaben entsprechend, bei $181-182^{\circ}$. Das specifische Gewicht der Base bei $20^{\circ}$ wurde zu 0,7902 gefunden.

Dieses Dimethylconiin erwies sich als stark rechtsdrehend, der Drehungswinkel wurde zu $13,47^{\circ}$ gefunden, während das Ausgangsmaterial einen Drehungswinkel von $9,8^{\circ}$ zeigte. Dies entspricht einem Drehungsvermögen

$$
\begin{array}{lrl}
\text { für Coniin } & {\left[\alpha_{\mathrm{D}}\right]=11, \mathrm{c} 6} \\
\text { fïr Dimethylconiin }\left[\alpha_{\mathrm{D}}\right]=17,04 .
\end{array}
$$

Wären wir vom reinen $\alpha$-Coniin ausgegangen, so hätten wir voraussichtlich für das Dimethylconiin ein Drehungsvermögen von über $20^{\circ}$ gefunden.

Schon oben ist ausgeführt worden, zu welchen Folgerungen diese bemerkenswerthe Thatsache führt. Die in Analogie mit dem Dimethylpiperidin zunächst wahrscheinliche Formel

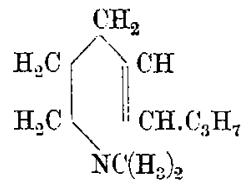

ist, da sie kein asymmetrisches Kohlenstoffatom enthält, nach der Le Bel-van t'Hoff'schen Theorie unmöglich.

Die dann zunächst in Betracht zu ziehende Formel<smiles>C=CCCC(CC)C(C)CCC</smiles>

erscheint aus verschiedenen Gründen unannehmbar. Sie constatirt eine Analogie zu dem Dimethylpiperidin, von dem es insofern ganz verschieden ist, als es durch Zerlegung mit Salzsäure keine Pyrrolidinverbindung erzeugt, sondern Coniin regenerirt, während die wirklich vorhandene Analogie mit dem Dimethylpipecolin durch diese Formel keinen Ausdruck erbält, da eine 
entsprechende Formel für diesen Körper aufzustellen unmöglich ist. All' dies soll im Folgenden näher begründet werden.

\section{Umuandlung von Dimethylconiin in Coniin.}

In Dimethylconiin wurde trockne Salzsïure so lange geleitet, bis die Gewichtszunahme zwei Molekülen Salzsäure entsprach, wozu eine T'emperatur von $100^{\circ}$ genügt. Es bildet sich so das salzsaure Hydrochlordimetbylconiin. Dieses wurde in Wasser gelöst, mit Aether überschichtet und unter líühlung durch vorsichtigen Zusatz von Natronlauge die Base abgeschieden, die sich sofort in dem Aether löst. Nach der Entfernung des letzteren bleibt ein Oel, das sich aber beim Erhitzen weder umwandelt, noch $\mathrm{CH}_{3} \mathrm{Cl}$ abspaltet.

Deshalb wurde bei einem zweiten Versuch das wie oben dargestellte salzsaure Hydrochlordimethylconiin weiter und höher unter Salzsäureeinleiten erhitzt. Erst bei $220^{\circ}$ beginnt eine langsame $\mathrm{CH}_{3} \mathrm{Cl}$-Entwickelung, die stundenlang andauert, und bei der nebenbei etwas Conylen extsteht. Nach beendeter Reaction wird in Wassor gelöst, von braunen Verumreinigungen durch Ausschütteln mit Aether befreit, uud der Rüclistand mit Natron destillirt. Die übergegangene Base wird mit hali abgeschieden und über Kali getrocknet. Bei der Destillation geht dieselbe zwischen $168^{\circ}$ und $178^{\circ}$ über und zwar die Hauptmenge zwischen $168^{\circ}$ und $170^{\circ}$. Da ein constanter Siedepunkt nicht zu erreichen war, so wurde die Base in Chlorhydrat verwandelt, das theiliveise krystallirt, theils syrupös zurückblieb. Die Krystalle wurden ron dem Syrup durch $\mathrm{Ab}$ saugen und Abpressen getrennt und dam mehrfach aus eirem Gemisch von Aceton und Methylalkobol umkrystallisirt. So wurden schliesslich aus $30 \mathrm{~g}$ Dimethylconiin $2 \mathrm{~g}$ ganz weisses, constant schmelzendes Chlorhydrat gewonnen, das der Analyse nach die Zusammensetzung des salzsauren Coniins besass. 
$0,0977 \mathrm{~g}$ gaben $0,2107 \mathrm{CO}_{2}$ und $0,0998 \mathrm{H}_{\mathrm{g}} \mathrm{O}$.

$\begin{array}{ccc} & \text { Berechnet für } & \text { Gefunden } \\ \text { c } & \mathrm{C}_{8} \mathrm{H}_{17} \mathrm{NHCl} & \\ \mathrm{H} & 58,70 & 58,80 \\ & 11,05 & 11,35\end{array}$

Auch soust zeigte das Salz vollständige Uebereinstimmung mit salzsaurem $\alpha$-Coniin: der Schmelzpunlat des Chlorhydrats liegt bei $218,5^{\circ}$, der Schmelzpunkt des Jodkadmiumsalzes bei $111-113^{\circ}$, das Platinsalz ist in Aetheralkohol vollständig löslich $\left.{ }^{13}\right)$.

Nach alledem bleibt für das Dimethylconiin nur die Formel<smiles>CCCC(CC)(CC)C(C)(C)CC</smiles>

Für die anderen Dimethylderivate von $a$-substituirten Piperidinen müssen ähnliche Formeln als wahrscheinlich angesehen werden und so erbält $z$. B. das Dimethylpipecolin folgende Formel:<smiles>CC(C)C1CCC2CCCC21</smiles>

Nach dieser Auffassung besitzt das Dimethylpipecolin ein asymmetrisches Kohlenstoffatom, und es muss also möglich sein, dasselbe in activer Form zu erhalten.

Die dahin zielenden Versuche lasse ich hier folgen.

VII. Umwandlung von 7-Pipecolin in actives Dimethylpipecolin.

$\mathrm{Zu}$ den folgenden Versuchen wurde ein mit $\mathrm{r}$-Pipecolin gemengtes 1-Pipecolin benutzt, wie man es als Nebenproduct

13) Vergl. auch La denburg, diese Annalen $\mathbf{2 4 7}, 1 \mathrm{ff}$. Dort ist der Schmelzpunkt des Jodkadmiumdoppelsalzes zu $117-118^{\circ}$ angegeben, doch darf diese kleine I)ifierenz wohl unberiicksichtigt bleibes. 
bei der Darstellung von $\boldsymbol{\kappa}$-Pipecolin erhält. Dasselbe zeigte im Decimeterrohre eine Drehung von

$$
\alpha_{\mathrm{D}}=-18,75^{\circ} \text {. }
$$

Die Umwandlung dieser Base in Dimethylderivat geschah genau so, wie es oben bei der Darstellung des Dimethylconiins beschrieben wurde, doch sei dabei bemerkt, dass das durch Jodmethyl gebildete Ammoniumjodür hier schwerer in Chloroform löslich ist, als die entsprechende Coniinverbindung und dass deshalb mehrfaches Auskochen mit diesem Lösungsmittel nothwendig ist.

Das so diargestellte Dimethylpipecolin siedet bei $142-143^{\circ}$ (Merling giebt $143-143,5^{\circ}$ an) und zeigt eine Drehung: von $-0,56^{\circ}$.

Da diese schwache Drehung der Vermuthung Raum gab, sie sei durch eine Verunreinigung der Base z. B. durch kleine Mengen von Methylpipecolin veranlasst, so ward die Ueberführung in Dimethylpipecolin nochmals wiederholt, nur ward jetzt die Zerlegung der Ammoniumbase im Vacuum vorgenommen, so dass bei der Destillation die Temperatur nicht über $110^{\circ}$ stieg, da es doch für sehr möglich angesehen wurde, dass bei der Destillation ein Theil der Base racemisch geworden war. Und in der That zeigte die jetzt gewonnene Base einen Drehungswinkel von $-2,63^{\circ}$.

Ich glaube daraus den Schluss ziehen zu sollen, dass das Dimetbylpipecolin selbst einen Einfluss auf den polarisirten Lichtstrahl ausübt und dass es deshalb ein asymmetrisches Kohlenstoffatom enthält. Wir kommen so für das Dimethylpipecolin zu einer ganz ähnlichen Formel wie für das Dimethylconiin, nämlich zu dem Schema<smiles>CC1CC2C1CC1CC2C(C)C1C</smiles> 
Es erscheint dann das Dimethylpipecolin als ein naher Verwandter zweier Basen, die kürzlich dargestellt wurden, nämlich des Pentamethenylamins<smiles>CC1(C)CCC1(C)N</smiles>

welches Wislicenus und Hentzschel durch Reduction des Adipinketoxims gewonnen haben und der $\beta$-Methylverbindung desselben, die Semmler in ähnlicher Weise aus dem $\beta$-Methylpentamethenylketoxim erhielt.

Leider ist das Verhalten dieser Basen, namentlich gegen Salzsäure, nicht näher studirt, um daraus einen Schluss ziehen zu können, ob diese ähnliche Auffassung auch einen thatsächlichen Untergrund hat.

Bemerkenswerth ist jedenfalls, dass diese ringförmig constituirten Basen, wie Dimethylpipecolin und Dimethylconiin, ebenso leicht Salzsäure addiren, wie das Dimethylpiperidin, in dem eine doppelte Bindung angenommen wird. Dasselbe gilt auch für das Verhalten gegen Jod.

Für das Dimethylpiperidin habe ich schon vor längerer Zeit nachgewiesen, dass es leicht zwei Atome Jod aufnimmt, dass das so entstehende Dijodür durch Chlorsilber ein Atom Jod gegen Chlor austauscht, in ein Chlorojodür übergeht, während durch Silberoxyd beide Jodatome entzogen werden und ein Oxydhydrat gebildet wird, das bei der trocknen Destillation Dimethylpiperideïn liefert. Aehnlich verbält sich Dimethylconiin gegen Jod.

VIII. Addition von Jod an Dimethylconiin und Verhalten des Dimethylconiinjodürs.

Setzt man zu einer Lösung von Dimethylconiin in absolutem Alkohol eine alkoholische ${ }^{14}$ ) Jodlösung, so verschwindet beim

14) Bei Anwendung von Schwefelkohlenstoff oder Chloroform als Lösungsmittel bildet sich viel harzige substanz. 
Umschütteln die Farbe derselben sehr rasch und es scheidet sich ein Krystallmehl ab. Man bört mit dem Zusatz von Jod auf, sobald die Flüssigkeit cine danernde Gelbfürbung annimmt, was weit ror dem Zusatz von zwei Atomen Jor stattfindet. Damn wird vou den ausgeschiedenen Krystallen abgesaugt und dieselben aus hcissem Wasser umlrrystallisirt, wobei sie als weisse Warzen erhalten werden, die nach dem Trockinen bei $184^{\circ}$ unter Zersetzung schmelzen. Die Aualyse zeigte, dass das erwartete Jodür $\mathrm{C}_{10} \mathrm{H}_{21} \mathrm{NJ}_{2}$ vorlag.

I. 0,1129 gralen $0,1227 \mathrm{CO}_{2}$ and $0,0527 \mathrm{H}_{2} \mathrm{O}$.

II. $0,0885 \mathrm{~g}$, nach $\mathrm{K} . \mathrm{j}$ e l d a l l l's Methode zerlegt, verbranchten $2,2 \mathrm{ccm}$ $1 / 10$ normal Salzsänre.

III. 0,1571 g, ebenso belandelt, $3,8 \mathrm{ccm}$, 10 norual Salzsäure.

Berechunet füir

Gefuuden $\mathrm{C}_{10} \mathrm{H}_{21} \mathrm{NJJ}_{2}$

$\begin{array}{rrrr}\mathrm{C} & 29,34 & 29,64 & - \\ \mathrm{H} & 5,11 & 5,19 & - \\ \mathrm{N} & 3,42 & 3,48 & 3,32\end{array}$

Wird das Jodür mit Chlorsilber geschüttelt, so bildet sich Jodsilber, und das Filtrat enthält ein Chlorojodür, das mit Platinchlorid ein schwer lösliches Salz bildet, welches aus heissem Wasser umkrystallisirt die erwartete Formel $\left(\mathrm{C}_{10} \mathrm{H}_{21} \mathrm{NJCl}\right)_{2} \mathrm{PtCl}_{4}$ besitzt.

$0,2229 \mathrm{~g}$ gaben $0,0450 \mathrm{Pt}$.

$\begin{array}{cc}\text { Berechuet } & \text { Gefunden } \\ 20,04 & 29,15\end{array}$

Die พässrige Lösung dieses Chlorojodürs reagirt auf Lakmus ganz neutral und bleibt beim Verdunsten krystallinisch zurück. Die concentrirte wässrige Lösung dreht die Polarisationsebene des Lichtes $i^{0}$ nach rechts, so dass an der Asymmetrie des Dimethylconiins kein Zweifel bleiben kanu.

Die Versuche, an das Dimcthylconiin Wasserstoff zu addiren, führten zu keinem jositiven Resultate, ebenso wenig wic frülnerc Versuche, welche ich bei dem Dimethylpiperidin ausgefüht hatte.

Nach alledem zeigt sich immerhin eine grosse Analogie zwischen Dimethylpiperidin einerseits and Dimethylpipecolin 
und Dimethylconiin andererseits, namentlich gilt dies hinsichtlich der Bildungsweise der drei Körper und auch in Bezug auf ihre Additionsfäligkeit von Salzsäure und Jod. Ilieser Analogie tragen offenbar die Formeln dieser Körper:

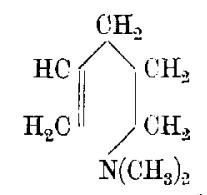

Dimethylpiperidin<smiles>CC(C)[C@H]1CC[C@H]2CCCC21</smiles>

Dimethylpipecolin<smiles>CCCC1CCCC1C(C)C</smiles>

Dimethylconiin

keine oder jedenfalls nur sehr unvollständig Rechnung und es erscheint daher die Frage berechtigt, ob wirklich die Theorie des asymmetrischen Kohlenstoffs so fest begründet erscheint, un derartige Folgerungen, wie sic hier gezogen wurden und die Autstellung obiger Formeln zu rechtfertigen. Meiner Ansicht nach muss diese Frage bejaht werden, da die angezogene Theorie schon so viele Erfolge anfzuweisen hat und mit so vielen Thatsachen in Uebereinstimmung stelt, dass dagegen die Unwahrscheinlichkeiten, die in der Deutung der hier entwickelten Thatsachen zu Tage treten, verschwinden.

Auders urtheilt Baeyer in eiwer kürzlich erschienenen Arbeit ${ }^{15}$ ). Dort kommt er zu dem Resultate, dass das Dipenten, welches die racemische Verbindung zweier enantiomorpher Substanzen ist, keinen asymmetrischen Kohlenstoff enthält und dass deshalb die optische Activität des Limonens auf einer Asymmetrie des Moleküls beruht, die nicht an das Vorkommen eines asymmetrischen Lohlenstoffatoms gebunden ist.

Ich muss gestehen, dass ich mit Baeyer's Schlussweise nicht einverstanden bin. Sie basirt in erster Linie auf der Identität zweier Tetrabromide, die namentlich durch die krystallographische Messung bewiesen werden soll. Die hier gegebenen Zahlen sind aber derart, dass sie die Möglichkeit einer Verschiedenheit nicht uubedingt ausschliessen ${ }^{16}$ ). Feruer handelt

15) Ber. d. dentsch. chem. Ges. 27, 436.

16) Aelnlich urtheilt College Hintze, wie aus Folgendem hervorgeht: Ich werde gefragt, ob ich nach den krystallographischen An- 
es sich namentlich um die Lage einer doppelten Bindung. Nun weiss man aber, und gerade zumeist durch Baeyer's Untersuchungen, wie leicht eine Verschiebung derselben eintreten kann. Und wem auch Baeyer ,eine Verschiebung der doppelten Bindung unter diesen Umständen tür unwahrscheinlich

gaben des Herrn Villiger (Ber. d. dentsch. chem. Ges. 1894, 27, 441) die Identität der von diesem gemessenen Krystalle mit den früher von mir (diese Aunalen 227, 279; Groth's Zeitschr. 10, 255) gemessenen Krystallen des Dipententetrabromids tür ,unzweifelhaft" halte. Diese Frage kamm ich nicht bejahen.

Gerade bei Terpenverbindungen ist mehrfach eine iusserliche Winkelähnlichkeit der Krystalle verschiedener Körper beobachtet worden. Ich erimnere au die Wink tälnnlichkeit zwischen Limonenund Dipententetrabromid (Groth's Zeitschr. 10, 256):

$$
\text { a : b : c c (100) (111) (010) (111) }
$$

Tetrabromid des Limonens $=0, \tilde{a} L: 1: 0,43 \quad 52^{\circ} 15^{\prime} \quad 71^{\circ} 52^{\prime}$ Tetrabromid des Dipentens $=0,52: 1: 0,45 \quad 51^{\circ} 57^{\prime} \quad 71^{\circ} 10^{\prime}$ andererseits zwischen Limonentetrabromid und Sesquiterpenhydrochlorid (Groth's Zeitschr. 13, 331):

$$
\begin{array}{ll}
C_{10} \mathrm{H}_{16} \mathrm{Br}_{4} & (122)(010)=68^{\circ} 30^{\prime} \\
c_{15} \mathrm{H}_{24} .2 \mathrm{H}(1 & (122)(010)=68^{\prime \prime} 34^{\prime}
\end{array}
$$

Bei diesen unter sich verschiedenen Krystallen ist doch offenbar eine noch grössere Cebereinstimmung der Winkel vorhanden, als

\begin{tabular}{|c|c|c|c|c|}
\hline & $\begin{array}{l}\text { Villiger } \\
\text { gemessen }\end{array}$ & $\begin{array}{l}\text { Villiger } \\
\text { berechmet }\end{array}$ & $\begin{array}{r}\text { Hintze } \\
\text { gemessen }\end{array}$ & $\begin{array}{l}\text { Hintzo } \\
\text { berechnet }\end{array}$ \\
\hline$(010)(140)$ & $=24^{\circ} 47^{\prime}:$ & - & $25^{\circ} 25^{\prime}$ & $25^{\circ} 31^{\prime}$ \\
\hline$(101)(140)$ & $=74^{\circ} 10^{\prime ; z}$ & $\ldots$ & - & $73^{\circ} 42^{\prime}$ \\
\hline (101) (101) & $=?$ & $81^{\circ} 13^{\prime}$ & $81^{\circ} 16^{\prime: *}$ & - \\
\hline 00) (111) & $=51^{0} 20^{\prime}$ & $52^{\prime \prime} \quad 8^{4}$ & $\check{5} 1^{0} \tilde{5} 3^{\prime}$ & $51^{\circ} 57^{\prime}$ \\
\hline 10) (111) & $=57^{\prime \prime} 42^{\prime}$ & $56^{0} \quad 1 / a^{\prime}$ & - & $56^{0} 10^{\prime}$ \\
\hline 10) (111) & $=?$ & $70^{\circ} 35^{\prime}$ & $71^{0} 10^{\prime}$ & $71^{\circ} 10^{\prime}$ \\
\hline
\end{tabular}
zwischen den von Herrn Villiger gemessenen Krystallen und den meinigen des Wallach'sehen Dipententetrabromids:

Gerade die von Herm Villiger als die besten der Rechnung zu Grunde gelegten Winkel (010) (140) und (101) (140) stimmen schlecht genng mit den entsprechenden des Dipententetrabromids. Da aber dem Zuviel des einen ein Zuwenig des anderen gegenübersteht, so muss der aus beiden resultirende Winkel (101) (101) naturgemäss 
hält", so muss ich gestehen, dass die subjective Ansicht eines Einzelnen doch verschwinden muss gegenüber einer Theorie, die, wie die in Rede stehende, eine solche Fülle von Thatsachen unter einen Gesichtspunkt zu bringen vermag.

Die Ansicht Baeyer's will mir um so weniger einleuchten, als Baeyer in keiner Weise angiebt oder auch nur andeutet, wie die Asymmetrie des Moleküls zu Stande kommen soll, wenn kein asymmetrisches Atom darin vorhanden sein soll; denn eine Asymmetrie des Wasserstoffs ist doch nicht denkbar, ebensowenig wie die Asymmetrie eines Moleküls, wenn dasselbe eine Symmetricebene besitzt und mit seinem Spiegelbilde identisch ist.

\section{Nachtrag.}

Während des Druckes dieser Ablandlung haben wir noch den Versuch gemacht, Dimethylpiperidin in zwei optisch active Verbindungen zu spalten. Wäre es wirklich möglich, Körper ohne asymmetrische Atome in optisch activer Form zu erhalten, so müsste ein solcher Versuch gerade bei dem Dimethylpiperidin aussichtsvoll sein, da dasselbe bestimmt kein asymmetrisches Kohlenstoffatom (und auch kein asymmetrisches Stickstoffatom)

einen Ausgrleich bieten und seine lebereinstimmung $\left(81^{\circ} 13^{\prime}\right.$ zu $81^{\circ} 16^{\prime}$ ) ist deshalb in diesem Falie von geringer Bedeutung für den Identitiitsberveis.

Ueber das optische Verhalten der Krystalle des Herru Villiger wird gar nichts gesagt, ebenso wenig iiber die Oberflichenbeschaffenlıeit und die l'enacität, die gerade für die Krystalle des Dipententetrabromids so sehr charaliteristisch und ein ganz rorzügliches Kennzeichen sind. Auch der Habitns der Krystalle des Herru Villiger scheint ein anderer gewesen zu sein (mit "pyramidaler Eudigung"), als der der meinigen (mit stets vorherrschend domatischer Endigung); das würde freilich noch nicht die Verschiedenheit beweisen, noch weniger aber die Identitüt.

Meines Erachtens steht also die Sache so: es ist wohl möglich, dass die von Herrn Villiger und von mir gemessenen Krystalle derselben Substanz angehören, "unzweifelhaft" bewiesen ist es nicht.
Bresla u, den 9. Mai 1894.
C. Hintze. 
enthält, und doch andererseits dem Dimethylconiin und dem Dimethylpipecolin, die beide optisch activ erhalten wurden, sehr nahe steht.

Es wurde deshalb reines Dimethylpiperidin in Bitartrat verwandelt und dieses zum Krystallisiren gebracht. Nachdem der auskrystallisirte Theil von der Mutterlauge getrennt worden war, blieb diese lange Zeit flüssig, erstarrte aber neulich doch. Die sorgfältig abgepressten Krystalle wurden zerlegt und die getrocknete Base optisch untersucht. Sie erwies sich als vollständig inactiv. Dieser Versuch entscheidet zwar nicht, spricht aber doch auch gegen v. Baeyer's Auffassung.

\section{Zur Kenntniss der Terpene und der ätherischen Oele; von 0 . Wallach.}

[Neunundzwanzigste AbbandIung.] (Mittheilung aus dem chemischen Institut der Universität Göttingen.)

I. Ueber Verbindungen der Carvonreihe. (Mitbearbeitet von H. Schrader.)

Obgleich bekanntlich die von H. Goldschmidt zuerst aufgestellte Carvon-Formel für den Fortschritt der Forschung die besten Dienste geleistet hat, existiren doch Thatsachen, welche sich mit ihr nur schlecht vereinigen lassen. Seit geraumer Zeit sind daher Untersuchungen über das Carvon von mir in Augriff genommen, welche bezwecken, jene Widersprüche zu beseitigen, beziehungsweise cine allen Thatsachen besser Rechnung tragende Carvon-Formel zu suchen.

Ueber einen Theil der bei diesen Arbeiten gewonnenen Resultate und zwar über denjenigen, welcher eine unmittelbare Fortsetzung der zuletzt über das Verhalten der cyclischen 